\title{
A predisposição genética para o desenvolvimento da microangiopatia no DM1: errata
}

Maria Lúcia Corrêa-Giannella, Suzana Maria Vieira

\section{Correção do Artigo}

A predisposição genética para o desenvolvimento da microangiopatia no DMl

Arq Bras Endocrinol Metab 2008;52(2):375-86

No oitavo parágrafo onde se lê:

Nesse estudo, a proporção de portadores de DMl que tinha ritmo de filtração glomerular (RFG) estimado pela fórmula de Cockroft-Gault $<60 \mathrm{~mL} / \mathrm{min} / 1,73 \mathrm{~m}^{2}$ e EUA normal $(<30 \mathrm{mg} / 24 \mathrm{~h})$ variou de $29 \%$ aos 5 a 6 anos de estudo até $52 \% \mathrm{em}$ l a 2 anos de estudo.

Leia-se:

Nesse estudo, a proporção de portadores de DMl que tinha ritmo de filtração glomerular (RFG) estimado pela fórmula derivada do estudo Modification of Diet in Renal Disease $(M D R D)<60 \mathrm{~mL} / \mathrm{min} / 1,73 \mathrm{~m}^{2}$ e EUA normal $(<30 \mathrm{mg} / 24 \mathrm{~h})$ variou de $29 \%$ aos 5 a 6 anos de estudo até $52 \%$ em 1 a 2 anos de estudo. 


\section{Habilidade de indicadores antropométricos e de composição corporal em identificar a resistência à insulina: errata}

Ana Carolina Junqueira Vasques, Lina Enriqueta Frandsen Paez de Lima Rosado,

Gilberto Paixão Rosado, Rita de Cassia Lanes Ribeiro, Sylvia do Carmo Castro

\section{Correção do Artigo}

Habilidade de indicadores antropométricos e de composição corporal em identificar a resistência à insulina

Arq Bras Endocrinol Metab 2009;53(1):72-79

Na Tabela 1 onde se lê:

Tabela 1. Indicadores antropométricos estudados para a identificação do risco de resistência à insulina.

\begin{tabular}{lcc}
\hline Indicadores & Fórmulas & Referências \\
\hline Índice de conicidade & $\mathrm{PC}(\mathrm{m}) / 0,109 \times \sqrt{\text { peso corporal }(\mathrm{kg})} /$ Estatura $(\mathrm{cm})$ & $(7)$ \\
Relação cintura/estatura & $\mathrm{PC}(\mathrm{cm}) /$ Estatura $(\mathrm{cm})$ & $(8)$ \\
Relação cintura/quadril & $\mathrm{PC}(\mathrm{cm}) / \mathrm{PQ}(\mathrm{cm})$ & (11) \\
Relação cintura/coxa & $\mathrm{PC}(\mathrm{cm}) / \mathrm{PCoxa}(\mathrm{cm})$ & $(12)$ \\
Índice sagital & $\mathrm{DAS}(\mathrm{cm}) / \mathrm{PCoxa}(\mathrm{cm})$ & $(15)$ \\
Índice de massa corporal & $\mathrm{Peso}(\mathrm{kg}) /$ Estatura $^{2}(\mathrm{~cm})$ & \\
\hline
\end{tabular}

$\mathrm{PC}=$ perímetro da cintura; $\mathrm{PQ}=$ perímetro do quadril; $\mathrm{PCoxa}=$ perímetro da coxa; $\mathrm{DAS}=$ diâmetro abdominal sagital.

Leia-se:

Tabela 1. Indicadores antropométricos estudados para a identificação do risco de resistência à insulina.

\begin{tabular}{lcc}
\hline Indicadores & Fórmulas & Referências \\
\hline Índice de conicidade & $P C(\mathrm{~m}) / 0,109 \times \sqrt{[\text { peso corporal }(\mathrm{kg}) / \text { Estatura }(\mathrm{m})]}$ & $(7)$ \\
Relação cintura/estatura & $P C(\mathrm{~cm}) /$ Estatura $(\mathrm{cm})$ & $(8)$ \\
Relação cintura/quadril & $\mathrm{PC}(\mathrm{cm}) / \mathrm{PQ}(\mathrm{cm})$ & $(11)$ \\
Relação cintura/coxa & $\mathrm{PC}(\mathrm{cm}) / \mathrm{PCoxa}(\mathrm{cm})$ & $(13)$ \\
Índice sagital & $\mathrm{DAS}(\mathrm{cm}) /$ PCoxa $(\mathrm{cm})$ & $(15)$ \\
Índice de massa corporal & $\mathrm{PeSo}(\mathrm{kg}) /$ Estatura $^{2}(\mathrm{~m})$ & \\
\hline
\end{tabular}

$P C=$ perímetro da cintura; $P Q=$ perímetro do quadril; $P C o x a=$ perímetro da coxa; $D A S=$ diâmetro abdominal sagital.

No tópico Análises bioquímicas onde se lê:

HOMA-IR $=\mathrm{IJ}(\mu \mathrm{U} / \mathrm{mL} \times \mathrm{GJ}(\mathrm{mmol} / \mathrm{L}) / 22,5$

Leia-se:

HOMA-IR $=\mathrm{IJ}(\mu \mathrm{U} / \mathrm{mL}) \times \mathrm{GJ}(\mathrm{mmol} / \mathrm{L}) / 22,5$ 